\title{
Some Implications of a Scale Invariant Model of Statistical Mechanics to Classical and Relativistic Thermodynamics
}

\author{
Siavash H. Sohrab \\ Robert McCormick School of Engineering and Applied Science \\ Department of Mechanical Engineering \\ Northwestern University, Evanston, Illinois 60208, USA \\ s-sohrab@northwestern.edu
}

\begin{abstract}
Some implications of a scale invariant model of statistical mechanics to the mechanical theory of heat of Helmholtz and Clausius are described. Modified invariant definitions of heat and entropy are presented closing the gap between radiation and gas theory. Modified relativistic transformations of pressure, Boltzmann constant, entropy, and density are introduced leading to transformation of ideal gas law. Following Helmholtz, the total thermal energy of thermodynamic system is decomposed into free heat $U$ and latent heat $\mathrm{p} V$ and identified as modified form of the first law of thermodynamics $Q=H=U+\mathrm{p} V$. Subjective versus objective aspects of Boltzmann thermodynamic entropy versus Shannon information entropy are discussed. Also, modified thermodynamic properties of ideal gas are presented. The relativistic thermodynamics being described is in accordance with Poincaré - Lorentz dynamic theory of relativity as opposed to Einstein kinematic theory of relativity since the former theory that is based on compressible ether of Planck is causal as was emphasized by Pauli.
\end{abstract}

Keywords: Relativistic thermodynamics; ideal gas; thermodynamics and information entropy; TOE

\section{Introduction}

Although the universality of statistical nature of problems of stochastic quantum fields [1-17] and classical hydrodynamic fields [18-30] is well known, the extent to which exact correspondence exists between the laws of nature amongst the diverse scales of space and time from cosmic to photonic schematically shown in Figure 1 is as yet not recognized. Similarities between the statistical fields shown in Figure 1 resulted in recent introduction of a scaleinvariant model of statistical mechanics [31] and its application to the fields of thermodynamics [32-34], fluid mechanics [35-38] and quantum mechanics [35, 39-40].

In the present study, further implications of the model to the physical foundations of classical and relativistic thermodynamics will be examined. The properties of ideal gas and subjective versus objective nature of entropy are investigated. Also, Helmholtz decomposition of thermal energy into free and latent heat is applied to define the nature of mass, dark matter, and dark energy.

\section{A Scale invariant model of statistical mechanics}

The scale-invariant model of statistical mechanics for equilibrium galactic-, planetary-, hydro-system-, fluidelement-, eddy-, cluster-, molecular-, atomic-, subatomic-, kromo-, and tachyon-dynamics corresponding to the scale $\beta=\mathrm{g}, \mathrm{p}, \mathrm{h}, \mathrm{f}, \mathrm{e}, \mathrm{c}, \mathrm{m}, \mathrm{a}, \mathrm{s}, \mathrm{k}$, and $\mathrm{t}$ is schematically shown on the left hand side of Figure 1. The corresponding nonequilibrium hence non-statistical laminar flow fields are shown on the right-hand-side of Figure 1. Each statistical field is identified as the "system" that is composed of an spectrum of "elements". Each element is composed of an ensemble of small particles called the "atoms" of the field that are viewed as point-mass and governed by distribution

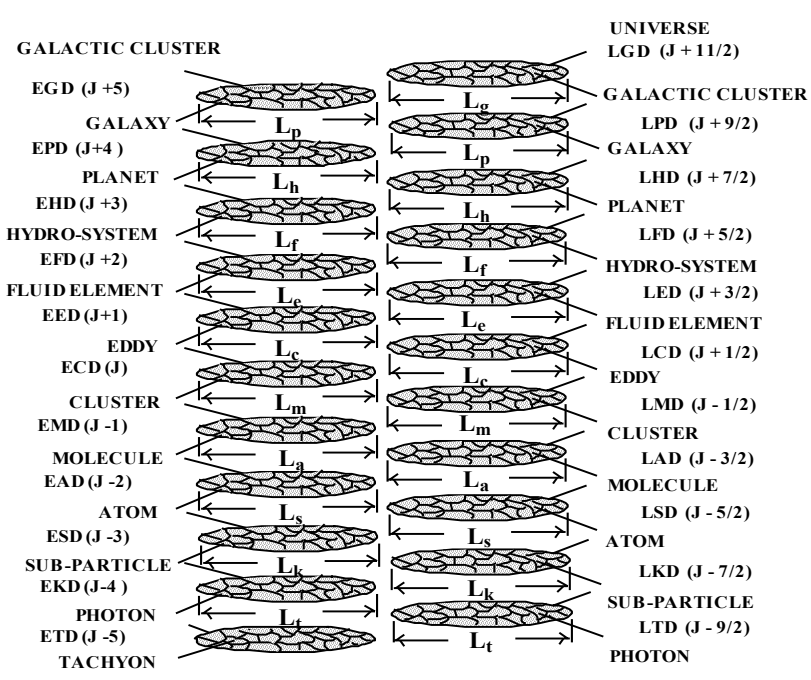

Figure 1. A Scale-invariant model of statistical mechanics. Equilibrium- $\beta$-Dynamics on the left-hand-side and nonequilibrium Laminar- $\beta$-Dynamics on the right-hand-side for scales $\beta=g, p, h, \mathrm{f}, e, c, m, a, s, k$, and $t$ as defined in Section 2. Characteristic lengths of (system, element, "atom”) are $\left(\mathrm{L}_{\beta}, \lambda_{\beta}, \ell_{\beta}\right)$ and $\lambda_{\beta}$ is the mean-free-path.

function $\int f_{\mathrm{i} \beta}\left(\mathbf{x}_{\mathrm{i} \beta}, \mathbf{u}_{\mathrm{i} \beta}, \mathrm{t}_{\beta}\right) \mathrm{d} \mathbf{x}_{\mathrm{i} \beta} \mathrm{d} \mathbf{u}_{\mathrm{i} \beta}$. The most probable element (system) velocity of the smaller scale $(\mathrm{J})$ becomes the velocity of the atom (element) of the larger scale (J+1). Since invariant Schrödinger equation was recently derived from invariant Bernoulli equation [39], the entire hierarchy of statistical fields shown in Figure 1 is governed by quantum mechanics. There are no physical or mathematical 
reasons for the hierarchy shown in Figure 1 not to continue to larger and smaller scales ad infinitum. Hence, according to Figure 1, contrary to the often quoted statement by Einstein that God does not play dice; the Almighty appears to be playing with infinite hierarchies of embedded dice.

Following the classical methods [19, 41-45] the invariant definition of density $\rho_{\beta}$, and velocity of atom $\mathbf{u}_{\beta}$, element $\mathbf{v}_{\beta}$, and system $\mathbf{w}_{\beta}$ (Figure 1 ) at the scale $\beta$ are [39, 40]

$$
\begin{array}{lll}
\rho_{\beta}=\mathrm{n}_{\beta} \mathrm{m}_{\beta}=\mathrm{m}_{\beta} \int f_{\beta} \mathrm{du} \mathrm{u}_{\beta} & , & \mathbf{u}_{\beta}=\mathbf{v}_{\mathrm{mp} \beta-1} \\
\mathbf{v}_{\beta}=\rho_{\beta}^{-1} \mathrm{~m}_{\beta} \int \mathbf{u}_{\beta} f_{\beta} \mathrm{du}_{\beta} & , & \mathbf{w}_{\beta}=\mathbf{v}_{\mathrm{mp} \beta+1}
\end{array}
$$

Similarly, the invariant definition of the peculiar and diffusion velocities are introduced as

$$
\mathbf{V}_{\beta}^{\prime}=\mathbf{u}_{\beta}-\mathbf{v}_{\beta} \quad, \quad \mathbf{V}_{\beta}=\mathbf{v}_{\beta}-\mathbf{w}_{\beta}
$$

such that

$$
\mathbf{V}_{\beta}=\mathbf{V}_{\beta+1}^{\prime}
$$

\section{Stochastic definitions of Planck and Boltzmann constants}

At thermodynamic equilibrium the mean velocity of particles, Heisenberg-Kramers virtual oscillators [46], vanish $<\mathrm{u}_{\mathrm{mx}}>=0$ such that the kinetic energy of particle oscillating in two directions $\left(\mathrm{x}_{+}, \mathrm{x}^{-}\right)$is expressed as

$$
\begin{aligned}
\varepsilon_{\beta}=\mathrm{m}_{\beta}\left\langle\mathrm{u}_{\beta \mathrm{x}+}^{2}\right\rangle / 2+\mathrm{m}_{\beta}\left\langle\mathrm{u}_{\beta \mathrm{x}-}^{2}\right\rangle / 2 \\
=\mathrm{m}_{\beta}\left\langle\mathrm{u}_{\beta \mathrm{x}+}^{2}\right\rangle=\overline{\mathrm{p}}_{\beta}\left\langle\lambda_{\beta}^{2}\right\rangle^{1 / 2}\left\langle v_{\beta}^{2}\right\rangle^{1 / 2}
\end{aligned}
$$

where $\bar{p}_{\beta}=m\left\langle u_{\beta x+}^{2}\right\rangle^{1 / 2}$ is the root-mean-square momentum of particle and $\left\langle\mathrm{u}_{\beta \mathrm{x}+}^{2}\right\rangle=\left\langle\mathrm{u}_{\beta \mathrm{x}-}^{2}\right\rangle$ by Boltzmann equipartition principle. At any scale $\beta$, the result in Eq. (5) can be expressed in terms of either frequency or wavelength

$$
\begin{aligned}
& \varepsilon_{\beta}=\mathrm{m}_{\beta}\left\langle\mathrm{u}_{\beta}^{2}\right\rangle=\overline{\mathrm{p}}_{\beta}\left\langle\lambda_{\beta}^{2}\right\rangle^{1 / 2}\left\langle v_{\beta}^{2}\right\rangle^{1 / 2}=\mathrm{h}_{\beta} v_{\beta} \\
& \varepsilon_{\beta}=\mathrm{m}_{\beta}\left\langle\mathrm{u}_{\beta}^{2}\right\rangle=\overline{\mathrm{p}}_{\beta}\left\langle v_{\beta}^{2}\right\rangle^{1 / 2}\left\langle\lambda_{\beta}^{2}\right\rangle^{1 / 2}=\mathrm{k}_{\beta} \lambda_{\beta}
\end{aligned}
$$

when the definition of stochastic Planck and Boltzmann factors are introduced as [35]

$$
\begin{aligned}
& \mathrm{h}_{\beta}=\overline{\mathrm{p}}_{\beta}\left\langle\lambda_{\beta}^{2}\right\rangle^{1 / 2} \\
& \mathrm{k}_{\beta}=\overline{\mathrm{p}}_{\beta}\left\langle\mathrm{v}_{\beta}^{2}\right\rangle^{1 / 2}
\end{aligned}
$$

At the important scale of EKD (Figure 1) corresponding to Casimir vacuum [47] composed of photon gas, the universal constants of Planck [48, 49] and Boltzmann [32] are identified from Eqs. (7a)-(7b) as

$$
\mathrm{h}=\mathrm{h}_{\mathrm{k}}=\mathrm{m}_{\mathrm{k}} \mathrm{c}\left\langle\lambda_{\mathrm{k}}^{2}\right\rangle^{1 / 2}=6.626 \times 10^{-34} \mathrm{~J}-\mathrm{s}
$$

$\mathrm{k}=\mathrm{k}_{\mathrm{k}}=\mathrm{m}_{\mathrm{k}} \mathrm{c}\left\langle v_{\mathrm{k}}^{2}\right\rangle^{1 / 2}=1.381 \times 10^{-23} \mathrm{~J} / \mathrm{K}$

Next, following de Broglie hypothesis for the wavelength of matter waves [2]

$\lambda_{\beta}=\mathrm{h} / \overline{\mathrm{p}}_{\beta}$

the frequency of matter waves is defined as [32]

$\mathrm{v}_{\beta}=\mathrm{k} / \overline{\mathrm{p}}_{\beta}$

When matter and radiation are in the state of thermodynamic equilibrium Eq. (8) leads to

$\mathrm{h}_{\beta}=\mathrm{h}_{\mathrm{k}}=\mathrm{h} \quad, \quad \mathrm{k}_{\beta}=\mathrm{k}_{\mathrm{k}}=\mathrm{k}$

The definitions in Eqs. (8a) and (8b) result in the gravitational mass of photon [32]

$\mathrm{m}_{\mathrm{k}}=\left(\mathrm{hk} / \mathrm{c}^{3}\right)^{1 / 2} \approx 1.84278 \times 10^{-41} \mathrm{~kg}$

that is much larger than the reported [50] value of $4 \times 10^{-51}$ $\mathrm{kg}$. The finite gravitational mass of photons was anticipated by Newton [51] and is in accordance with Einstein-de Broglie theory of light [52-56]. Avogardo-Loschmidt number was predicted as [32]

$\mathrm{N}^{\mathrm{o}}=1 /\left(\mathrm{m}_{\mathrm{k}} \mathrm{c}^{2}\right) \approx 6.0376 \times 10^{23}$

leading to the modified value of the universal gas constant

$\mathrm{R}^{\circ}=\mathrm{N}^{\mathrm{o}} \mathrm{k} \approx 8.338 \mathrm{~kJ} /(\mathrm{kmol}-\mathrm{K})$

Also, by Eq. (13) the atomic mass unit becomes

$$
\begin{aligned}
\mathrm{amu}= & \mathrm{m}_{\mathrm{k}} \mathrm{c}^{2} \\
& =(\mathrm{hkc})^{1 / 2} \approx 1.6563 \times 10^{-27} \mathrm{~kg} / \mathrm{kmol}
\end{aligned}
$$

Since all baryonic matter is known to be composed of atoms, Eqs. (12) and (15) suggest that all matter in the universe is composed of light [33]. By Eqs. (8a)-(8b) the wavelength and frequency of photon in vacuum $\left\langle\lambda_{k}^{2}\right\rangle^{1 / 2}\left\langle v_{k}^{2}\right\rangle^{1 / 2}=c$ are

$$
\begin{aligned}
\lambda_{\mathrm{k}}=\left\langle\lambda_{\mathrm{k}}^{2}\right\rangle^{1 / 2}= & 1 / \mathrm{R}^{\mathrm{o}} \approx 0.119935 \\
v_{\mathrm{k}} & =\left\langle v_{\mathrm{k}}^{2}\right\rangle^{1 / 2} \approx 2.49969 \times 10^{9} \mathrm{~Hz}
\end{aligned}
$$

\section{Modified definitions of thermodynamic temperature and pressure}

The classical definition of thermodynamic temperature is based on three translational degrees of freedom such that

$3 \mathrm{kT}^{\prime}=\mathrm{mv^{2 }}=\mathrm{m}\left(\overline{\mathrm{v}_{\mathrm{x}}^{2}}+\overline{\mathrm{v}_{\mathrm{y}}^{2}}+\overline{\mathrm{v}_{\mathrm{z}}^{2}}\right)=\mathrm{mv}_{\mathrm{t}}^{2}$

One may have two possible interpretation of the above formula. First is that there are translational kinetic energy 
due to simultaneous motion in $(\mathrm{x}, \mathrm{y}, \mathrm{z})$ coordinate directions which is physically impossible. In reality translational motion of particle only takes place in one arbitrary direction, say $\mathrm{x}$. Therefore, a second interpretation of Eq. (17) is that the translational energy of motion along a single arbitrary direction is decomposed into three independent coordinate directions. The assumption of isotropy of space leads to Boltzmann principle of equipartition of energy and hence $\varepsilon_{\mathrm{t} 1}=\varepsilon_{\mathrm{t} 2}=\varepsilon_{\mathrm{t} 3}$.

According to Eq. (3), particle translational velocity is the sum of the mean or cluster velocity and the random peculiar velocity

$$
\mathbf{u}_{\mathrm{mj}}=\mathbf{v}_{\mathrm{mj}}+\mathbf{V}_{\mathrm{mj}}^{\prime}=\mathbf{u}_{\mathrm{cj}}+\mathbf{V}_{\mathrm{mj}}^{\prime}
$$

The above definition is in the same spirit as in cosmology where the peculiar velocity of a galaxy is defined as the difference between its velocity and the mean velocity of the cluster of galaxies to which it belongs. The thermodynamic system being considered herein is composed of a spectrum of molecular clusters under stochastically stationary state. In a recent study [39], it was shown that three different flow regimes based on the nature of cluster velocity $\mathbf{v}_{\mathrm{m}}$ in Eq. (3) could be identified. For a system of ideal gas at thermodynamic equilibrium all three velocities in Eq. (18) are random such that Eq. (18) when squared, averaged, and multiplied by particle mass leads to the kinetic energy

$\overline{m u_{m x j}^{2}}=m \overline{v_{m x j}^{2}}+m \overline{V_{m x j}^{\prime 2}}=m \overline{u_{c x j}^{2}}+m \overline{V_{m x j}^{\prime 2}}$

since $\overline{\mathbf{u}_{\mathrm{cxj}} \mathbf{V}_{\mathrm{mxj}}^{\prime}}=0$. First the internal energy of particle due to translational motion of "cluster" in two directions $\left(\mathrm{X}^{+}, \mathrm{X}^{-}\right)$is expressed as

$$
\begin{aligned}
\hat{u}_{t}=\frac{1}{2} \mathrm{mv}_{\mathrm{mx}+}^{2} & +\frac{1}{2} \overline{\mathrm{mv}_{\mathrm{mx}-}^{2}}= \\
& =\frac{1}{2} \overline{\mathrm{mu}_{\mathrm{cx}+}^{2}}+\frac{1}{2} \overline{\mathrm{mu}_{\mathrm{cx}-}^{2}}=\mathrm{mv}_{\mathrm{mx}+}^{2}
\end{aligned}
$$

Next, the potential energy due to "stress" is related to the kinetic energy of peculiar velocity as

$$
\begin{aligned}
& \hat{\varepsilon}_{p}=\frac{1}{2} \mathrm{~m}_{\mathrm{V}_{\mathrm{mx}+}^{\prime 2}}+\frac{1}{2} \mathrm{~m}_{\overline{\mathrm{V}_{\mathrm{mx}-}^{\prime 2}}}= \\
& =\mathrm{m} \overline{V_{m x+}^{\prime 2}}=\frac{1}{3} \mathrm{mV}_{\mathrm{m}+}^{\prime 2}=p \hat{v}
\end{aligned}
$$

where pressure is defined as $\mathrm{p}=\mathrm{nmV}_{\mathrm{m}+}^{\prime 2} / 3$. Hence, the total energy associated with harmonic translational motion of particle will become

$$
\hat{\varepsilon}_{\mathrm{t}}=\hat{u}_{t}+\hat{\varepsilon}_{p}=\hat{u}_{t}+\mathrm{p} \hat{\mathrm{v}}
$$

Clausius in his investigation of the mechanical theory of heat emphasized that the energy due to rotation and vibration of particles are significant [57]

"In liquids, therefore, an oscillatory, a rotatory, and a translator motion of the molecules take place, but in such a manner that these molecules are not themselves separated from each other, but even in the absence of external forces, remain within a certain volume"

Thus far the history of physics has shown that there are no such things as absolutely rigid elementary particles. Therefore, following Clausius the internal kinetic energy of rotational and vibrational motion of particles in two directions are written as [58]

$$
\begin{aligned}
& \hat{u}_{r}=\varepsilon_{r}=\frac{1}{2} \overline{I \omega_{\mathrm{m} \theta+}^{2}}+\frac{1}{2} \overline{I \omega_{\mathrm{m} \theta-}^{2}}=I \overline{\omega_{\mathrm{m} \theta+}^{2}} \\
& \hat{u}_{v}=\varepsilon_{v}=\frac{1}{2} \chi \overline{\mathrm{r}_{\mathrm{m}+}^{2}}+\frac{1}{2} \chi \overline{\mathrm{r}_{\mathrm{m}-}^{2}}=\chi \overline{\mathrm{r}_{\mathrm{m}+}^{2}}
\end{aligned}
$$

where $(I, \chi)$ are respectively the moment of inertia and the spring constant. The dissipation of translational, rotational, and vibrational (pulsational) motions are schematically shown in Figure 2.

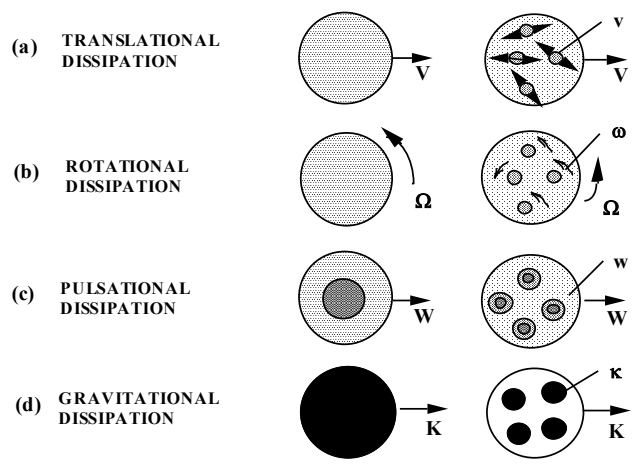

Figure 2. Dissipation of energy from global $(\mathbf{V}, \mathbf{\Omega}, \mathbf{W}, \mathbf{K})$ to local $(\mathbf{v}, \mathbf{\omega}, \mathbf{w}, \mathbf{\kappa})$ : (a) translational (b) rotational (c) vibrational (d) gravitational degrees of freedom.

According to Figure 2, energy of global average translational, rotational, and vibrational motions could be dissipated into the corresponding local random motions thus being "transformed" to what Helmholtz called free heat. The last dissipation mechanism (d) of Figure 2 refers to dissipation of scalar curvature and hence will be ultimately connected to what is referred to as gravitational viscosity [59, 40].

Following the classical methods, the internal "atomic" energy of particle is defined as the sum of its translational, rotational, and vibrational kinetic energy from eqs. (20), (23), and (24)

$\hat{u}=\hat{u}_{t}+\hat{u}_{r}+\hat{u}_{v}=\overline{\mathrm{m} \overline{\mathrm{x}}^{2}}+I \overline{\omega_{\theta+}^{2}}+\chi \overline{\mathrm{r}_{+}^{2}}$

Finally, by Eqs. (22) and (25) the total atomic energy or atomic enthalpy is defined as the sum of internal kinetic energy and external potential energy and written as [40] 
$\hat{h}=\hat{u}_{t}+\hat{u}_{r}+\hat{u}_{v}+\mathrm{p} \hat{\mathrm{v}}=\hat{u}+\mathrm{p} \hat{\mathrm{v}}$

such that the total enthalpy becomes

$H=U+\mathrm{p} V$

where $(H, U, V)=\mathrm{N}(\hat{h}, \hat{u}, \hat{\mathrm{v}})$.

According to Eq. (26) the system has four degrees of freedom and at equilibrium Boltzmann principle of equipartition of energy requires

$\hat{u}_{t}=\hat{u}_{r}=\hat{u}_{v}=\mathrm{p} \hat{\mathrm{v}}$

Also, equipartition principle of Boltzmann in Eq. (28a) can be extended to include all translational degrees of freedom such that

$\hat{u}_{t}=\hat{u}_{\mathrm{x}}=\hat{u}_{\mathrm{y}}=\hat{u}_{\mathrm{z}}=\mathrm{p} \hat{\mathrm{v}}$

that is in accordance with the virial theorem of Clausius [60].

Since harmonic translational oscillations are considered to occur in two directions $(\mathrm{x}+, \mathrm{x}-),(\mathrm{y}+, \mathrm{y}-),(\mathrm{z}+, \mathrm{z}-), \mathrm{Eq}$. (17) becomes

$$
\begin{aligned}
3 \mathrm{kT}^{\prime}=2 \mathrm{~m}\left(\overline{\mathrm{v}_{\mathrm{x}+}^{2}}+\overline{\mathrm{v}_{\mathrm{y}+}^{2}}+\overline{\mathrm{v}_{\mathrm{z}+}^{2}}\right)= \\
=2\left(\hat{u}_{x}+\hat{u}_{y}+\hat{u}_{z}\right)=2 \hat{u}
\end{aligned}
$$

If one attributes the rotational and vibrational kinetic energy $\left(\hat{u}_{\mathrm{r}}, \hat{u}_{\mathrm{v}}\right)$ to the kinetic energy of translational motions $\left(\hat{u}_{\mathrm{y}}, \hat{u}_{\mathrm{z}}\right)$ along the coordinates $(\mathrm{y}, \mathrm{z})$ the classical form of internal energy in Eq. (29a) and the modified form in Eq. (25) become equivalent with the exception of a factor of 2 due to inclusion of motion in two directions $(x+$, $\mathrm{x}-)$ according to the modified theory. Hence, one can express Eq. (29a) as

$$
3 \mathrm{kT}^{\prime}=\mathrm{mv}_{\mathrm{t}}^{2}=\mathrm{mv}_{\mathrm{t+}}^{2}+\mathrm{mv}_{\mathrm{t}-}^{2}=2 \mathrm{mv}_{\mathrm{t+}}^{2}=2 \hat{u}
$$

leading to the classical expression for the internal energy

$U^{\prime}=\frac{3}{2} \mathrm{NkT}^{\prime}$

Recently, a modified definition of thermodynamic temperature $\mathrm{T}$ based on two translational degrees of freedom was introduced as [40]

$$
3 \mathrm{kT}=\mathrm{mv}_{\mathrm{t}+}^{2}=\hat{u}_{t}+\hat{u}_{r}+\hat{u}_{v}=\hat{u}
$$

that results in the modified form of internal energy

$$
U=3 \mathrm{NkT}
$$

As a result, the classical and modified definitions of temperature and pressure are related by
$\mathrm{T}^{\prime}=2 \mathrm{~T} \quad, \quad \mathrm{p}^{\prime}=2 \mathrm{p}$

Although the equivalence of $\left(\hat{u}_{\mathrm{r}}, \hat{u}_{\mathrm{v}}\right)$ and $\left(\hat{u}_{\mathrm{y}}, \hat{u}_{\mathrm{z}}\right)$ leads to identical particle internal energy it is suggested herein that the classical model does not correspond to the true motion of particles. This is because in reality each particle can physically move only in one arbitrary translational coordinate direction at any instant of time. Therefore, only translational, rotational, and vibrational motions can constitute three simultaneous independent degrees of freedom. It could be argued that according to the classical practice all energy is attributed to a single arbitrary translational degree of freedom and Eq. (29a) merely represents a vector decomposition of this total translational kinetic energy into three independent coordinate directions due to principle of equipartition of energy. However, while isotropy of space does require stochastic equipartition of energy along any arbitrary direction clearly this does not mean presence of actual physical motion along such coordinate directions. For example, if one considers harmonic translation of a particle confined to a spherical vessel, space isotropy requires that for time durations much longer than the period of oscillations particle should touch the surface area of the vessel homogeneously. Also, as argued above and according to Clausius [57] the rotational and vibrational kinetic energy of particles are significant and cannot be properly neglected.

The factor 2 in Eq. (33) results in the predicted speed of sound $a$ in standard air $[58,61]$

$$
\begin{aligned}
a= & \mathrm{v}_{\text {max }}=\sqrt{\mathrm{p} / \rho}=\sqrt{\mathrm{p}^{\prime} /(2 \rho)} \\
& =\sqrt{3 \mathrm{kT}^{\prime} /(2 \mathrm{~m})}=\sqrt{3 \mathrm{R}^{\circ} \mathrm{T}^{\prime} / 2 \tilde{\mathrm{W}}} \approx 358 \mathrm{~m} / \mathrm{s}
\end{aligned}
$$

in close agreement with observations. Also, Eq. (34) leads to calculated r.m.s. molecular speeds $(1365,341,365,305$, $965,291) \mathrm{m} / \mathrm{s}$ that are in reasonable agreement with the observed velocities of sound $(1286,332,337,308,972$, 268) $\mathrm{m} / \mathrm{s}$ in $\left(\mathrm{H}_{2}, \mathrm{O}_{2}, \mathrm{~N}_{2}, \mathrm{Ar}, \mathrm{He}, \mathrm{CO}_{2}\right)$ at standard temperature [61].

The square root of 2 in Eq. (34) resolves the classical problem of Newton concerning his prediction of velocity of sound as

$$
a^{\prime}=\sqrt{\mathrm{p}^{\prime} / \rho}
$$

discussed by Chandrasekhar [62]

"Newton must have been baffled, not to say disappointed. Search as he might, he could find no flaw in his theoretical framework-neither could Euler, Lagrange, and Laplace; nor, indeed, anyone down to the present"

Indeed, the expression introduced by Euler $p=\rho v^{2} / 3$, Lagrange $p \propto \rho^{4 / 3}$ as well as Laplace's assumption of isentropic relation $\mathrm{p}=\mathrm{b} \rho^{\gamma}$, where $\mathrm{b}$ is a constant and $\gamma=c_{p} / c_{v}$, that leads to the conventional expression for the speed of sound in ideal gas 


$$
\tilde{a}=\sqrt{\gamma \mathrm{RT}^{\prime}}
$$

where $\mathrm{R}=\mathrm{R}^{\circ} / \tilde{\mathrm{W}}$ and $\tilde{\mathrm{W}}$ is molecular weight, are all found to deviate from the experimental data as described by Brush [63].

For example, the predictions of the speed of sound in Helium and Argonne (He, Ar) with $\gamma=(1.667,1.667)$ based on classical theory in Eq. (36) versus the modified theory in Eq. (34) are

$$
\begin{aligned}
& \tilde{a}=\sqrt{\gamma \mathrm{kT}^{\prime} / \mathrm{m}}=(1018,322) \mathrm{m} / \mathrm{s} \\
& a=\sqrt{3 \mathrm{kT}^{\prime} /(2 \mathrm{~m})}=(965,305) \mathrm{m} / \mathrm{s}
\end{aligned}
$$

to be compared with the measured values [58]

$$
a=(972,308) \mathrm{m} / \mathrm{s}
$$

Although predictions of Eq. (37a) based on isentropic process are reasonable those of the modified theory in Eq. (37b) based on isothermal process are closer to the observed values given in Eq. (37c).

\section{The First law of thermodynamics and mechanical equivalent of heat}

Some of the implications of the result of the previous section to the first law of thermodynamics for a closed system composed of ideal gas and mechanical equivalent of heat are examined in this section. It is first noted that both Helmholtz [64] and Clausius [57, 60]) based the foundation of the first law of thermodynamics on the application of the virial theorem to a system composed of weakly interacting gas.

Historically, in his pioneering 1847 paper concerning the first law of thermodynamics Helmholtz argued that the thermal energy of the system may be decomposed into two parts, namely the kinetic energy or vis viva of particles and their potential energy due to the stress caused by their interactions [64]

"That which has been heretofore named the quantity of heat, according to this, be the expression, first, of the quantity of vis viva of the caloric motion, and secondly, of the quantity of those tensions between the atoms, which, by changing the arrangement of the latter, such a motion can develop. The first portion would correspond to that which has been heretofore called free heat, the second with that which has been named latent heat"

Therefore, following Helmholtz [64] the first law of thermodynamics is expressed as

\section{System thermal energy $=$ Free heat + Laten heat}

Hence, if one identifies $U$ and $\mathrm{p} V$ as free heat and latent heat respectively enthalpy becomes the total thermal energy of the system $H=Q$ and Eq. (27) may be viewed as the modified form of the first law of thermodynamics

$Q_{\beta}=U_{\beta}+W_{\beta}$
In Eq. (39) the reversible heat and reversible work are both state functions and defined as $[32,40]$

$Q=\mathrm{T} S \quad$ Reversible heat

$W=\mathrm{p} V \quad$ Reversible work

Eq. (39) is only valid for ideal gas at equilibrium $G=\mathrm{d} G=$ 0 and for non-ideal or non-equilibrium systems in the presence of "chemical" reactions it must include Gibbs chemical potential and becomes Euler equation [40]

$Q=\mathrm{T} S=U+W-\sum_{\mathrm{j}} \hat{\mu}_{\mathrm{j}} \mathrm{N}_{\mathrm{j}}=H-G$

where $\hat{\mu}_{\mathrm{i}}=\hat{g}_{\mathrm{i}}$ is chemical potential.

The system energy in Eq. (39) is not to be associated with $U$ but rather with the enthalpy $Q=H$. The potential energy or work $W$ in Eq. (39) through dissipation becomes also "thermal" in nature and relates to the kinetic energy due to random peculiar motion of particles by Eq. (21). When external thermal energy $\mathrm{d} Q$ is added to the system or mechanical work $-\mathrm{d} W$ is done by the system Eq. (39) relates the changes of the three state functions by

$d Q=d U+d W$

As a result, the paradox as to how the path-dependence of heat and work exactly cancel each other according to classical thermodynamics

$f(\delta Q-\delta W)=0$

is now resolved because both reversible heat and reversible work are state functions. This weakness of the classical formulation of the first law of thermodynamics is significant. This is because heat relates to microscopic random motions whereas work relates to macroscopic ordered motions such that the exact cancellation of their path-dependence is indeed paradoxical.

By Eq. (40) heat can be decomposed as

$d Q=\mathrm{T} d S+S \mathrm{dT}=d Q_{\mathrm{T}}+d Q_{\mathrm{S}}$

involving isothermal $d Q_{\mathrm{T}}$ and isentropic $d Q_{\mathrm{S}}$ heat. Decomposition of work from Eq. (41) is

$d W=\mathrm{p} d V+V \mathrm{dp}=d W_{\mathrm{p}}+d W_{\mathrm{v}}$

that involves isobaric (dilatational) work $d W_{\mathrm{p}}$ and isochoric (pressure or stress) work $d W_{\mathrm{v}}$.

For equilibrium condition at constant temperature and constant pressure by Eqs. (39), (45) and (46) the first law of thermodynamics becomes

$d Q_{\mathrm{T}}=d U+d W_{\mathrm{p}} \quad, \quad \mathrm{dT}=\mathrm{dp}=0$

and involves the conventional dilatational work 


$$
W_{\mathrm{p}}=\int \mathrm{p} d V=\mathrm{p}\left(V_{2}-V_{1}\right)
$$

Similarly, under equilibrium condition at constant temperature and constant volume by Eqs. (39), (45) and (46) the first law of thermodynamics becomes

$d Q_{\mathrm{T}}=d U+d W_{\mathrm{v}} \quad, \quad \mathrm{dT}=d V=0$

and involves pressure work such as the work done by a pump on incompressible fluid

$W_{\mathrm{v}}=\int V \mathrm{dp}=V\left(\mathrm{p}_{2}-\mathrm{p}_{1}\right)$

In classical thermodynamics the isochoric work defined above is neglected and in the analysis of pumps such work called "flow work" is related to the change of fluid enthalpy. More general implications of the new paradigm require further future investigations.

Next, the impact of the above results on mechanical equivalent of heat is examined. The factor of 2 in Eq. (33) also leads to the modified value of Joule-Mayer mechanical equivalent of heat $\mathbf{J}$ [39]

$$
\mathrm{J}=2 \mathrm{~J}_{\mathrm{c}}=2 \times 4.169=8338 \text { Joule } / \mathrm{kcal}
$$

where the value $J_{c}=4.169 \square 4.17[\mathrm{~kJ} / \mathrm{kcal}]$ is the average of the two values $J_{c}=(4.15,4.19)$ reported by Pauli [65]. The number in Eq. (51) is thus identified as the universal gas constant in Eq. (14) when expressed in appropriate MKS system of units

$$
\mathrm{R}^{\mathrm{o}}=\mathrm{kN}^{\mathrm{o}}=\mathrm{J}=8338 \text { Joule } /(\mathrm{kmol} \mathrm{K})
$$

The modified value of the universal gas constant in Eq. (52) was recently identified [66] as De Pretto number 8338 that appeared in the mass-energy equivalence equation of De Pretto [67]

$$
E=\mathrm{mc}^{2} \quad \text { Joule }=\mathrm{mc}^{2} / 8338 \mathrm{kcal}
$$

Unfortunately, the name of Olinto De Pretto in the history of evolution of mass energy equivalence is little known. Ironically, Einstein's best friend Michele Besso was a relative and close friend of Olinto De Pretto's brother Augusto De Pretto. The relativistic form of Eq. (53) was first introduced by Poincaré [68]

$$
E=\mathrm{m}_{\mathrm{r}} \mathrm{c}^{2}
$$

where $\mathrm{m}_{\mathrm{r}}=\mathrm{m}_{\mathrm{o}} / \sqrt{1-\mathrm{v}^{2} / \mathrm{c}^{2}}$ is Lorentz relativistic mass. Since the formula (53) is the only equation in the paper by De Pretto [67], the exact method by which he arrived at the number 8338 is not known even though one possible method was recently suggested [66]. The important contributions by Hasenöhrl [69] and Einstein [70] as well as the equivalence principle, equivalence of the rest or gravitational mass and the inertial mass were discussed in a recent study [33].

\section{Thermodynamic properties of ideal gas}

Before a critical comparison between entropy as defined in thermodynamics versus information theory is made, the exact nature of entropy for a simple system namely an ideal gas will be presented. The partition function $\mathrm{Z}$ and Helmholtz free energy $F$ of an ideal gas are given by Kardar [71] as

$\mathrm{Z}(\mathrm{T}, V, \mathrm{~N})=\frac{V^{\mathrm{N}}}{\mathrm{N} !}\left(\frac{2 \pi \mathrm{mkT}}{\mathrm{h}^{2}}\right)^{3 \mathrm{~N} / 2}=\frac{1}{\mathrm{~N} !}\left(\frac{V}{\lambda^{3}}\right)^{\mathrm{N}}$

and

$F=-\mathrm{NkT}\left[\ln \left(\frac{V \mathrm{e}}{\mathrm{N}}\right)+\frac{3}{2} \ln \left(\frac{2 \pi \mathrm{mkT}}{\mathrm{h}^{2}}\right)\right]$

with the characteristic wavelength defined as

$\lambda=\mathrm{h} / \sqrt{2 \pi \mathrm{mkT}}$

According to Eq. (8a) the characteristic wavelength associated with Planck constant $\mathrm{h}$ is de Broglie wavelength of matter waves in Eq. (9).

If one assumes that for an ideal gas at thermodynamic equilibrium the atomic volume $\hat{v}$ is precisely defined in terms of particle de Broglie wavelength in Eq. (9) as

$\hat{\mathrm{v}}=\lambda^{3}$

the volumetric number density of particles becomes

$\mathrm{n}=\frac{\mathrm{N}}{V}=\frac{1}{\lambda^{3}} \quad, \quad \frac{V}{\lambda^{3}}=\frac{V}{\hat{\mathrm{v}}}=\mathrm{N}$

Therefore, for ideal gas at a given temperature and volume $(T, V)$ the number of particles, Heisenberg-Kramers virtual oscillators [46] is given by

$\begin{cases}\hat{\mathrm{v}}>\lambda^{3} & \mathrm{~N}<\mathrm{N}_{\mathrm{cq}} \\ \hat{\mathrm{v}}=\lambda^{3} & \mathrm{~N}=\mathrm{N}_{\mathrm{eq}} \\ \hat{\mathrm{v}}<\lambda^{3} & \mathrm{~N}>\mathrm{N}_{\mathrm{eq}}\end{cases}$

In other words, at equilibrium for a given temperature $\mathrm{T}$ Eq. (9) gives de Broglie matter wavelength $\lambda$ such that atomic $\lambda^{3}$ and system $V$ volumes by Eqs. (58)-(59) lead to the equality

$\lambda^{3} \mathrm{n}=1$

Substituting from Eqs. (58)-(59) into Eq. (55) gives the partition function for ideal gas

$\mathrm{Z}=\frac{\mathrm{N}^{\mathrm{N}}}{\mathrm{N} !}=\mathrm{W} \approx \frac{\mathrm{N}^{\mathrm{N}}}{(\mathrm{N} / \mathrm{e})^{\mathrm{N}}}=\mathrm{e}^{\mathrm{N}}$

With equations (58) and (62) one obtains Helmholtz free energy for an ideal gas from Eq. (56) as

$F=-\mathrm{NkT}$ 
By substitution for Helmholtz free energy from Eq. (63) and in view of Eq. (58) the chemical potential of ideal gas at equilibrium becomes

$$
\hat{\mu}=\left.\frac{\partial F}{\partial \mathrm{N}}\right|_{\mathrm{T}, V}=\frac{F}{\mathrm{~N}}+\mathrm{kT}=\mathrm{kT} \ln \left(\mathrm{n} \lambda^{3}\right)=0
$$

The vanishing of Gibbs chemical potential $\hat{\mu}=\hat{g}=0$ for an ideal gas in Eq. (64) establishes an exact correspondence between ideal gas and photon gas thus closing the gap between radiation and gas theories [40]. This correspondence suggests that like photons the ideal gas system energy only weakly depends on the number of oscillators because of their exceedingly large numbers, about $10^{26}$ per one kmol meaning $10^{13}$ average-size clusters each containing $10^{13}$ particles. Since each cluster can break up into many smaller ones just like photons the number of Heisenberg-Kramers [46] virtual oscillators is practically infinite. One notes that just like photons non-conservation of number of oscillators of ideal gas means vanishing of the second Lagrange multiplier and hence chemical potential in the classical derivation of Boltzmann distribution [40, 72].

For non-ideal or multicomponent mixtures that are "reactive" the chemical potential will be finite and Eq. (64) assumes its classical form [72]

$$
\hat{\mu}_{\mathrm{i}}=\mathrm{kT} \ln \left[\lambda^{3} \mathrm{n}_{\mathrm{o}}\left(\frac{\mathrm{n}_{\mathrm{i}}}{\mathrm{n}_{\mathrm{o}}}\right)\right]=\hat{\mu}_{\mathrm{i}}^{\mathrm{o}}+\mathrm{kT} \ln \left(\frac{\mathrm{p}_{\mathrm{i}}}{\mathrm{p}_{\mathrm{o}}}\right)
$$

Hence, at a given (T, $V$ ) if the equilibrium criteria (60) and hence (64) are not satisfied such that $\mathrm{N}>\mathrm{N}_{\mathrm{eq}}\left(\mathrm{N}<\mathrm{N}_{\mathrm{eq}}\right)$, then the number of Heisenberg-Kramers virtual oscillators will decrease (increase) through changes in cluster sizes until the state of equilibrium is achieved and Gibbs free energy is minimized $d G=0$.

Next, entropy of ideal gas is determined directly from Eqs. (63) and (32) and the definition of Helmholtz free energy

$$
F=U-\mathrm{T} S=3 \mathrm{NkT}-\mathrm{T} S=-\mathrm{NkT}=-\mathrm{p} V
$$

as

$$
S=4 \mathrm{Nk}
$$

in exact agreement with the recent results obtained for photon gas [40]. The origin of the number 4 in Eq. (67) may be connected to the total number of complexions $\overline{\mathrm{W}}$ associated with four independent degrees of freedom namely translational, rotational, vibrational, and potential and defined as

$$
\overline{\mathrm{W}}=\mathrm{W}_{\mathrm{t}} \cdot \mathrm{W}_{\mathrm{r}} \cdot \mathrm{W}_{\mathrm{v}} \cdot \mathrm{W}_{\mathrm{p}}=\mathrm{W}^{4}
$$

Substituting from Eq. (68) into Eq. (85) of the next section gives the total number of complexions [34]

$$
\overline{\mathrm{W}}=\left(\frac{\mathrm{N}^{\mathrm{N}}}{\mathrm{N} !}\right)^{4} \approx \mathrm{e}^{4 \mathrm{~N}}
$$

that by Boltzmann Eq. (84) gives the same result as in Eq. (67).

At the end of this section some implications of what we call Helmholtz decomposition of thermal energy into free versus latent heat is examined. For example, it is possible to introduce a modified form of Boltzmann thermodynamic entropy in Eq. (84) starting from Boltzmann distribution function [40] expressed in normalized form as

$\mathrm{N}_{\mathrm{j}}=\mathrm{Ne}^{-\left(H_{\mathrm{j}}-\hat{\mu}\right) / \mathrm{kT}}$

where the energy $\left(E-E_{\mathrm{j}}\right)$ is identified as transition enthalpy $H_{\mathrm{j}}$ that by Eq. (32) and for an ideal gas $\mathrm{p}_{\mathrm{j}} V=\mathrm{N}_{\mathrm{j}} \mathrm{kT}$ becomes

$H_{\mathrm{j}}=U_{\mathrm{j}}+\mathrm{p}_{\mathrm{j}} V=4 \mathrm{~N}_{\mathrm{j}} \mathrm{kT}$

Next, by equations (64) and (71) Eq. (70) gives

$\mathrm{N}_{\mathrm{j}}=\mathrm{Ne}^{-H_{\mathrm{j}} / \mathrm{kT}}=\mathrm{Ne}^{-4 \mathrm{~N}}$

thus leading to the definition of probability of state $\mathrm{j}$

$p_{\mathrm{j}}=\mathrm{N}_{\mathrm{j}} / \mathrm{N}=\mathrm{e}^{-4 \mathrm{~N}_{\mathrm{j}}}$

The alternative form of Boltzmann thermodynamic entropy is now introduced as

$S_{\mathrm{j}}=-\mathrm{k} \ln p_{\mathrm{j}}$

Substitution from Eq. (73) into Eq. (74) gives

$S_{\mathrm{j}}=4 \mathrm{kN}_{\mathrm{j}}$

in exact agreement with Eq. (67). The reason for equivalence of Boltzmann theory in Eq. (84) versus the modified theory in Eq. (74) is that Boltzmann distribution in Eq. (70) is itself derived on the basis of maximization of $\mathrm{W}[40,72]$.

The result in Eq. (67) differs from the entropy of an ideal gas according to Sackur-Tetrode formula [73]

$S^{\prime}=\mathrm{Nk}\left[\ln \left(\frac{V}{\mathrm{~N}}\right)+\frac{3}{2} \ln \left(\frac{2 \pi \mathrm{mkT}^{\prime}}{\mathrm{h}^{3}}\right)+\frac{5}{2}\right]$

One can express Eq. (76) as

$$
S^{\prime}=\mathrm{Nk}\left[\ln \left(\frac{V \mathrm{e}}{\mathrm{N}}\right)+\frac{3}{2} \ln \left(\frac{2 \pi \mathrm{mkT}^{\prime}}{\mathrm{h}^{3}}\right)+\frac{3}{2}\right]
$$

that after substitutions from Eqs. (57)-(59) reduces to

$S^{\prime}=\mathrm{Nk}\left(1+\frac{3}{2}\right)=\frac{5}{2} \mathrm{Nk}$

Also, substituting the result in Eq. (78) into the expression for chemical potential gives 
$\frac{\hat{\mu}}{\mathrm{T}}=\left.\frac{\partial S^{\prime}}{\partial \mathrm{N}}\right|_{\mathrm{U}, \mathrm{V}}=-\frac{S^{\prime}}{\mathrm{N}}+\frac{5}{2} \mathrm{k}=0$

in agreement with Eq. (64).

However, when the modified definition of temperature in Eq. (33) that changes the factor $3 / 2$ to 3 in the internal energy $U$ according to Eq. (32) is taken into account the result in Eq. (78) becomes identical to that in Eq. (67) and Eq. (79) is modified as

$$
\frac{\hat{\mu}}{\mathrm{T}}=\left.\frac{\partial S}{\partial \mathrm{N}}\right|_{\mathrm{U}, \mathrm{V}}=-\frac{S}{\mathrm{~N}}+4 \mathrm{k}=0
$$

in accordance with the equilibrium criteria

$$
\mathrm{N} \hat{\mu}=G=-\mathrm{T} S+4 \mathrm{NkT}=-\mathrm{T} S+H=0
$$

Finally, from Eq. (63) the thermodynamic pressure becomes

$$
\mathrm{p}=-\left.\frac{\partial F}{\partial \mathrm{T}}\right|_{\mathrm{T}, \mathrm{N}}=\frac{\mathrm{NkT}}{V}
$$

that is the ideal gas law.

\section{Objective versus subjective nature of thermodynamic entropy}

Possible subjective versus objective nature of entropy has been subject of much debate ever since 1948 when Shannon $[74,75]$ used the name entropy in his information theory. The objective nature of thermodynamic entropy is evident from its classical definition first introduced by Clausius

$$
d S=d Q_{\mathrm{rev}} / \mathrm{T}
$$

In other words, direct connection between entropy $S$ and heat $Q$ clearly establishes the objective nature of the former.

The misunderstandings concerning possible subjective nature of entropy is in part because of its statistical definition first introduced by Boltzmann. [76, 77]

$$
S=\mathrm{k} \ln \mathrm{W} \quad, \quad S_{\mathrm{j}}=\mathrm{k} \ln \mathrm{W}_{\mathrm{j}}
$$

The total number of complexions is

$$
\mathrm{W}=\prod_{\mathrm{j}} \mathrm{W}_{\mathrm{j}}
$$

and the number of complexions for distributing $\mathrm{N}_{\mathrm{j}}$ indistinguishable particles among $\mathrm{g}_{\mathrm{j}}$ distinguishable cluster [40] or "quantum state" $\mathrm{j}$ according to combinatoric methods [78] of Boltzmann [76, 77] and Planck [49] is

$$
\mathrm{W}_{\mathrm{j}}=\frac{\left(\mathrm{N}_{\mathrm{j}}+\mathrm{g}_{\mathrm{j}}-1\right) !}{\mathrm{N}_{\mathrm{j}} !\left(\mathrm{g}_{\mathrm{j}}-1\right) !}
$$

If following the ideas of Onnes and Ehrenfest the quantity $\left(\mathrm{N}_{\mathrm{j}}+\mathrm{g}_{\mathrm{j}}-1\right)$ is viewed as the total number of symbols composed of $\mathrm{N}_{\mathrm{j}}$ indistinguishable particles and $\left(g_{j}-1\right)$ available distinguishable clusters one may consider the inverse of Eq. (86) as probability of complexion $\mathrm{j}$ [34]

$p_{\mathrm{j}}=\frac{\mathrm{N}_{\mathrm{j}} !\left(\mathrm{g}_{\mathrm{j}}-1\right) !}{\left(\mathrm{N}_{\mathrm{j}}+\mathrm{g}_{\mathrm{j}}-1\right) !}=\frac{1}{\mathrm{~W}_{\mathrm{j}}}$

such that Eq. (84) could be expressed as

$S=-\mathrm{k} \ln p \quad, \quad S_{\mathrm{j}}=-\mathrm{k} \ln p_{\mathrm{j}}$

when probability of all complexions are independent of one another and hence

$p=\prod_{\mathrm{j}} p_{\mathrm{j}}=\frac{1}{\mathrm{~W}}$

According to the information theory of Shannon [74, 75] the amount of information $\mathrm{H}$ also referred to as information entropy is expressed by Shannon formula

$\mathrm{H}=-K \sum_{\mathrm{j}} p_{\mathrm{j}} \ln p_{\mathrm{j}}$

where $K$ is a positive constant. On the other hand, thermodynamic entropy of mixture is obtained from Eqs. (88)-(89) as [34]

$S=\sum_{\mathrm{j}} \mathrm{N}_{\mathrm{j}} S_{\mathrm{j}}=-\mathrm{k} \sum_{\mathrm{j}} \mathrm{N}_{\mathrm{j}} \ln p_{\mathrm{j}}$

leading to "mixture" entropy [34] per particle

$S / \mathrm{N}=s=-\mathrm{k} \sum_{\mathrm{j}} p_{\mathrm{j}} \ln p_{\mathrm{j}}$

where $p_{\mathrm{j}}=\mathrm{N}_{\mathrm{j}} / \mathrm{N}$. Changes in information could be viewed as changes in entropy in harmony with ideas of Brillouin [79]. Equations (90) and (92) only differ by Shannon constant $K$ versus Boltzmann constant k.

The occurrence of the universal constant $\mathrm{k}$ in Eq. (92) has fundamental significance [34]. According to Equations (7)-(11) Planck and Boltzmann constants are two fundamental constants of nature that relate to spatial and temporal aspect of Casimir [47] vacuum fluctuations. Because all conceivable information must be transmitted by some physical entity, such as electron, photon, neutrino, etc. in space-time, it is expected that both constants (h, k) will play a central role in transmission of information. Therefore, it is not advisable to modify thermodynamic entropy in Eq. (92) to achieve correspondence with Eq. (90). Instead, choosing Shannon "measure" as $K=\mathrm{k}$ leads to exact equivalence of Eqs. (90) and (92) as discussed in [34]. Such equivalence can also be achieved by defining dimensionless Shannon information entropy and Boltzmann thermodynamic entropy as 


$$
\mathrm{H} / K=s / \mathrm{k}=-\sum_{\mathrm{j}} p_{\mathrm{j}} \ln p_{\mathrm{j}}
$$

Recently, it was suggested by Ben-Naim [80] that to achieve exact correspondence between Boltzmann entropy in thermodynamics in Eq. (92) and Shannon entropy in information theory in Eq. (90) the dimension of thermodynamics absolute temperature be changed [80]

\section{"One should redefine a new absolute temperature; denote it tentatively as $\overline{\mathbf{T}}$ to replace $k T$. The new temperature $\overline{\mathbf{T}}$ would have the units of energy and there would be no need for Boltzmann constant. The equation for entropy will be simply $S=\ln W,{ }^{21}$ and entropy would be rendered dimensionless"; "This will automatically expunge the Boltzmann constant $k_{B}$ from the vocabulary of physics"}

Some of the reasons as to why such a change of dimension of absolute thermodynamic temperature is not advisable are discussed in the sequel.

Equations (4)-(10) clearly show that the universal constants $(\mathrm{h}, \mathrm{k})$ are intimately related to the energy of stochastically stationary fluctuations of Casimir [47] vacuum with the dimensions

$$
\begin{array}{ll}
\varepsilon_{\beta}=\mathrm{h}\left\langle v_{\beta}^{2}\right\rangle^{1 / 2}=\mathrm{h}_{\beta} v_{\beta} & {[\mathrm{J}-\mathrm{s}] \cdot[\mathrm{Hz}]=[\mathrm{J}]} \\
\varepsilon_{\beta}=\mathrm{k}\left\langle\lambda_{\beta}^{2}\right\rangle^{1 / 2}=\mathrm{k}_{\beta} \lambda_{\beta} & {[\mathrm{J} / \mathrm{m}] .[\mathrm{m}]=[\mathrm{J}]}
\end{array}
$$

Therefore, neither Planck constant h nor what he defined as Boltzmann constant $\mathrm{k}$ could be expunged from the vocabulary of physics. Also, according to Eqs. (6) and (10) Kelvin absolute temperature is identified as a length scale associated with mean wavelength of thermal oscillations

$\varepsilon_{\beta}=\mathrm{k}\left\langle\lambda_{\beta}^{2}\right\rangle^{1 / 2}=\mathrm{kT}$

The identification of dimension of absolute thermodynamic temperature as [meter] rather than [degree $\mathrm{K}$ ] is a step towards clarification of the exact physical nature of this intensive property.

Because temperature is a measured quantity ultimately it is the theory that must determine the significance of such a variable and its dimension according to Planck [81].

"Every measurement first acquires its meaning for physical science through the significance which a theory gives it"

Therefore, following Planck's suggestion, it is reasonable to change the dimension of absolute thermodynamic temperature $T$ from [degree $\mathrm{K}$ ] to [meter]. The exact correspondence between $\mathrm{H}$ and $s$ could be achieved by dimensionless entropy in Eq. (93) or the choice of Shannon measure as $K=\mathrm{k}$ [34].

Arguments have also been made that the very name entropy first coined by its discoverer Clausius should be changed [82]

"It is also time to change not only the units of entropy to make it dimensionless, but the term "entropy" altogether. Entropy, as is now recognized, does not mean "transformation, or "change" or "turn". It does mean information. Why not replace the term that means "nothing" as Cooper noted, and does not even convey the meaning it was meant to convey when selected by Clausius? Why not replace it with a simple, familiar, meaningful, and precisely defined term "information?"

It is emphasized however that contrary to the above quotation the name entropy has been chosen most appropriately by Clausius to represent precisely what it should namely "transformation". According to the result in Eq. (67) for ideal gas, entropy relates to the number of Planck [48] or Heisenberg-Kramers [46] oscillators. Therefore, the second law of thermodynamics suggests that in all natural processes there is a tendency to transform energy and hence motion from ordered macroscopic motions of a few large-scale oscillators to random microscopic motions of many small-scale oscillators thus increasing the entropy of the system. In other words entropy according to its macroscopic definition by Clausius is directly related to heat

$d S=\frac{d Q_{\mathrm{rev}}}{\mathrm{T}}=\frac{d Q_{\mathrm{T}}}{\mathrm{T}}$

Therefore, all types of energy dissipation due to plastic deformation, friction, viscosity, etc. will lead to transformation of energy of ordered (correlated) motions into that of disordered (uncorrelated) random motions that is heat thus leading to increase of entropy by Eq. (96). It seems that if we follow the changes of dimensions and notations suggested by Ben-Naim [80, 82] the state of our scientific terminology may become void and hence approach what he calls "Tohu Vavohu" [80] meaning total chaos.

\section{The foundation of relativistic thermodynamics}

In this section some of the implications of the dynamic theory of relativity of Poincaré-Lorentz as opposed to kinematic theory of relativity of Einstein [39] to relativistic thermodynamics will be examined. Historically, Planck [83], Mosengeil [84], Einstein [85], Laue [86], and Jüttner [87] first established the foundation of relativistic thermodynamics through the introduction of the relativistic transformations of thermodynamic quantities

$$
\begin{aligned}
& Q=Q_{o} \sqrt{1-\mathrm{v}^{2} / \mathrm{c}^{2}} \\
& \mathrm{~T}=\mathrm{T}_{\mathrm{o}} \sqrt{1-\mathrm{v}^{2} / \mathrm{c}^{2}} \\
& \mathrm{p}=\mathrm{p}_{\mathrm{o}} \\
& S=S_{\mathrm{o}}
\end{aligned}
$$

The transformation of heat in Eq. (97) was also maintained by both de Broglie [3] as well as Pauli [88].

Because reversible heat is defined by Clausius as $\mathrm{d} Q_{\text {rev }}$ $=\mathrm{Td} S$, on the basis of Carnot cycle Kelvin arrived at the equality of ratios of heat and absolute temperature

$Q / Q_{\mathrm{o}}={ }^{?} \mathrm{~T} / \mathrm{T}$ 。 
Historically the satisfaction of Kelvin formula (101) was perhaps the main motivation behind the choice of relativistic transformations (97) and (98). However, one expects the transformation for heat $Q$ that is energy to follow Poincaré-Einstein relativistic energy transformation [68, 89-91]

$$
E=E_{\mathrm{o}} / \sqrt{1-\mathrm{v}^{2} / \mathrm{c}^{2}}
$$

In 1952 Einstein in his correspondence with Laue [92] challenged the relativistic transformations for heat and temperature in Eqs. (97) and (98) and proposed instead the modified formulas

$$
\begin{aligned}
& Q=Q_{\mathrm{o}} / \sqrt{1-\mathrm{v}^{2} / \mathrm{c}^{2}} \\
& \mathrm{~T}=\mathrm{T}_{\mathrm{o}} / \sqrt{1-\mathrm{v}^{2} / \mathrm{c}^{2}}
\end{aligned}
$$

The discrepancy between Eqs. (97)-(98) and (103)-(104) resulted in some major controversies concerning the foundation of relativistic thermodynamics [93-96]. In fact, recently Landsberg and Matsas [95] questioned the very existence of a continuous relativistic temperature transformation.

Because of the definition of Boltzmann constant in Eq. (8b) by Eq. (6) the absolute thermodynamic temperature is identified as a length scale

$\mathrm{T}_{\beta}=\left\langle\lambda_{\beta}^{2}\right\rangle^{1 / 2}$

Since wavelengths transform by the Lorentz-FitzGerald contraction [97] as

$<\lambda^{2}>^{1 / 2}=<\lambda_{0}^{2}>^{1 / 2} \sqrt{1-v^{2} / c^{2}}$

by Eqs. (105) and (106) one obtains

$$
\mathrm{T}=\mathrm{T}_{\mathrm{o}} \sqrt{1-\mathrm{v}^{2} / \mathrm{c}^{2}}
$$

that is in accordance with Planck formula (98) rather than Einstein formula (104).

The transformations for heat and temperature in Eqs. (13) and (107) are not compatible with Kelvin formula (101). To address this, one requires relativistic transformation of Boltzmann constant k. The stochastic definition of Boltzmann constant from Eq. (10) could be written as

$$
\mathrm{k}=\mathrm{mc}\left\langle v_{\mathrm{k}}^{2}\right\rangle^{1 / 2}=\mathrm{mc}^{2} /\left\langle\lambda_{\mathrm{k}}^{2}\right\rangle^{1 / 2}
$$

By the transformation of wavelengths in Eq. (106) and the Lorentz relativistic mass [97]

$$
\mathrm{m}=\mathrm{m}_{\mathrm{o}} / \sqrt{1-\mathrm{v}^{2} / \mathrm{c}^{2}}
$$

one obtains from Eq. (108) the transformation for the Boltzmann constant

$$
\mathrm{k}=\mathrm{k}_{\mathrm{o}} /\left(1-\mathrm{v}^{2} / \mathrm{c}^{2}\right)
$$

It is interesting that recently Avramov [98] anticipated the fact that the Boltzmann constant is not relativistically invariant and suggested the transformation

$$
\left(\mathrm{k}_{\mathrm{B}}\right)_{\mathrm{u}}=\left(\mathrm{k}_{\mathrm{B}}\right)_{\mathrm{o}} \sqrt{1-\mathrm{v}^{2} / \mathrm{c}^{2}}
$$

that does not agree with the formula (110) of the present theory. However, only by the formula (110) does Eq. (107) lead to the consistent energy transformation

$\mathrm{kT}=\varepsilon=\frac{\mathrm{k}_{\mathrm{o}} \mathrm{T}_{\mathrm{o}}}{\sqrt{1-\mathrm{v}^{2} / \mathrm{c}^{2}}}=\frac{\varepsilon_{\mathrm{o}}}{\sqrt{1-\mathrm{v}^{2} / \mathrm{c}^{2}}}$

By the expressions for reversible heat $Q=\mathrm{T} S$ in Eq. (40) and entropy $S=4 \mathrm{Nk}$ in Eq. (67) and the transformations of $\mathrm{T}$ and $Q$ in Eqs. (107) and (103) Kelvin formula (101) becomes

$$
\frac{Q}{Q_{o}}=\frac{4 \mathrm{NkT}}{4 \mathrm{~N}_{\mathrm{o}} \mathrm{k}_{\mathrm{o}} \mathrm{T}_{o}}=\frac{\mathrm{kT}}{\mathrm{k}_{\mathrm{o}} \mathrm{T}_{\mathrm{o}}}=\frac{\varepsilon}{\varepsilon_{o}}
$$

Therefore, a consistent relationship between Kelvin formula (101) and the transformation of $Q$ and $\mathrm{T}$ is only possible through the inclusion of the correct transformation of Boltzmann constant in Eq. (110).

Next, from the stochastic definition of Planck constant in Eq. (8a)

$$
\mathrm{h}=\mathrm{m}_{\mathrm{k}} \mathrm{c}\left\langle\lambda_{\mathrm{k}}^{2}\right\rangle^{1 / 2}
$$

and the transformations of wavelength in Eq. (106) and Lorentz relativistic mass in Eq. (109) one obtains relativistic invariance of Planck constant

$\mathrm{h}=\mathrm{h}_{\mathrm{o}}$

The invariance of Planck universal constant and the Planck formula for energy quanta $\varepsilon=h \nu$ along with the energy transformation

$\mathrm{h} v=\varepsilon=\frac{\mathrm{h}_{\mathrm{o}} v_{\mathrm{o}}}{\sqrt{1-\mathrm{v}^{2} / \mathrm{c}^{2}}}=\frac{\varepsilon_{\mathrm{o}}}{\sqrt{1-\mathrm{v}^{2} / \mathrm{c}^{2}}}$

lead to the frequency transformation

$v=v_{0} / \sqrt{1-v^{2} / c^{2}}$

The frequency dilatation in Eq. (117) leads to contraction of time duration or period $\tau$ as

$$
\tau=\tau_{\mathrm{o}} \sqrt{1-\mathrm{v}^{2} / \mathrm{c}^{2}}
$$

Thus, space extensions and time durations both contract by Eqs. (106) and (118) such as to maintain a constant velocity of light

$\lambda / \tau=\lambda_{\mathrm{o}} / \tau_{\mathrm{o}}=\mathrm{c}$ 
Therefore, the terminology "time dilatation" often used in physics literature is misleading since time durations actually contract due to motion. It is also important to note that strictly speaking the speed of light is not a constant but varies with the temperature of Casimir [47] vacuum as the universe expands. However, since the expansion of cosmos occurs through eons the velocity of light appears as a constant for time durations relevant to human civilization.

In accordance with Eq. (102), all energy related thermodynamic parameters $\mathrm{f}=\left(Q, H, U, W, G, F, \boldsymbol{p}, \mathrm{m}, \mathrm{R}^{\mathrm{o}}\right.$, $\mathrm{R}, \overline{\mathrm{N}}$ ) transform as

$$
\mathrm{f}=\mathrm{f}_{\mathrm{o}} / \sqrt{1-\mathrm{v}^{2} / \mathrm{c}^{2}}
$$

where $\left(\boldsymbol{p}=\mathrm{mv}, \quad \mathrm{R}^{\circ}, \quad \mathrm{R}=\mathrm{R}^{\circ} / \tilde{\mathrm{W}}, \overline{\mathrm{N}}=\mathrm{N} / \mathrm{N}^{\circ}\right)$ are momentum, universal gas constant, gas constant, number of moles, and $\tilde{W}$ is the molecular weight. Since physical space or Aristotle's ether is considered to be compressible in accordance with Planck's compressible ether [39-40], volume contracts according to the Lorentz-FitzGerald contraction [97]

$$
V=V_{\mathrm{o}} \sqrt{1-\mathrm{v}^{2} / \mathrm{c}^{2}}
$$

Also, whereas energy per particle follows Eq. (102)

$$
\hat{\varepsilon}=\hat{\varepsilon}_{\mathrm{o}} / \sqrt{1-\mathrm{v}^{2} / \mathrm{c}^{2}}
$$

energy per unit mass $\varepsilon$ and energy per unit volume $\tilde{\varepsilon}$ transform as

$$
\varepsilon=\varepsilon_{\mathrm{o}} \quad, \quad \tilde{\varepsilon}=\tilde{\varepsilon}_{\mathrm{o}} /\left(1-\mathrm{v}^{2} / \mathrm{c}^{2}\right)
$$

From the ideal gas law and the definition of reversible work in Eq. (41) the potential energy

$$
W=\mathrm{p} V=\mathrm{NkT}
$$

must transform by Eq. (120) and due to volume transformation in Eq. (121) pressure transforms as

$$
\mathrm{p}=\mathrm{p}_{\mathrm{o}} /\left(1-\mathrm{v}^{2} / \mathrm{c}^{2}\right)
$$

thus modifying the classical result in Eq. (99).

The transformations of volume and pressure by Eqs. (121) and (125) lead to density and specific volume transformations

$$
\rho=\rho_{\mathrm{o}} /\left(1-\mathrm{v}^{2} / \mathrm{c}^{2}\right) \quad, \quad \tilde{\mathrm{v}}=\tilde{\mathrm{v}}_{\mathrm{o}}\left(1-\mathrm{v}^{2} / \mathrm{c}^{2}\right)
$$

Hence, higher local densities will be measured by an observer moving with respect to a compressible medium as schematically shown in Figure 3.

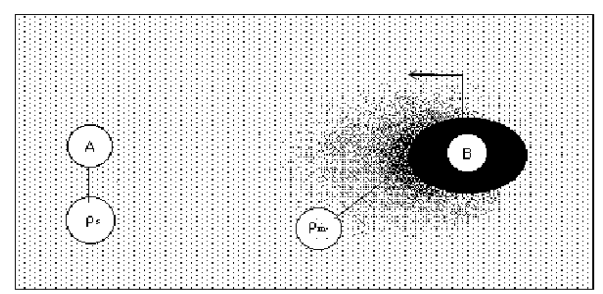

Figure 3. Density of a medium as measured by an observer that is (A) stationary (B) moving with respect to the medium.

In view of transformations for temperature and Boltzmann constant in Eqs. (107) and (110) entropy transforms as

$S=S_{\mathrm{o}} /\left(1-\mathrm{v}^{2} / \mathrm{c}^{2}\right)$

that modifies the classical result in Eq. (100). Also, the transformations in Eqs. (110) and (127) and Boltzmann formula (84) result in relativistic invariance of the number of complexions

$\mathrm{W}=\mathrm{W}_{\mathrm{o}}$

Finally, the transformations for Avogadro-Loschmidt number $\mathrm{N}^{\mathrm{o}}=1 / \mathrm{amu}$ and molecular weights $\tilde{\mathrm{W}}=\mathrm{mN}^{\mathrm{o}}$ are

$$
\begin{aligned}
& \mathrm{N}^{\mathrm{o}}=\mathrm{N}_{\mathrm{o}}^{\mathrm{o}} \sqrt{1-\mathrm{v}^{2} / \mathrm{c}^{2}} \\
& \tilde{\mathrm{W}}=\tilde{\mathrm{W}}_{\mathrm{o}}
\end{aligned}
$$

Recently, it was shown [40] that the invariant form of the first law of thermodynamics for ideal gas hence Eqs. (39), (41) and (67) also apply to photon gas or the radiation field within which it resides

$\mathrm{T} S=4 \mathrm{NkT}=U+\mathrm{p} V=3 \mathrm{NkT}+\mathrm{NkT}(131)$

Therefore, closure of the gap between gas theory and radiation theory [40] results in identical Helmholtz decomposition of thermal energy for ideal gas

Ideal $\begin{cases}U=\frac{3}{4} \mathrm{~T} S=3 \mathrm{NkT} & \text { Free heat } \\ \mathrm{p} V=\frac{1}{4} \mathrm{~T} S=\mathrm{NkT} & \text { Latent heat }\end{cases}$

as well as photon gas

Photon $\begin{cases}U=\frac{3}{4} \mathrm{~T} S=3 \mathrm{NkT} & , \lambda>\lambda_{\mathrm{w}} \\ \mathrm{p} V=\frac{1}{4} \mathrm{~T} S=\mathrm{NkT} & , \lambda<\lambda_{\mathrm{w}}\end{cases}$

It is known that exactly $3 / 4$ and $1 / 4$ of the total spectral energy of Planck black body or equilibrium radiation fall on $\lambda>\lambda_{w}$ 
and $\lambda<\lambda_{\mathrm{w}}$ sides of Wien wavelength $\lambda_{\mathrm{w}}$ given by Wien displacement law [40]

$$
\lambda_{\mathrm{w}} \mathrm{T}=\frac{0.2014}{\mathrm{R}^{\mathrm{o} 2}}=0.002897 \mathrm{~m} . \mathrm{K}
$$

in agreement with Eq. (132b). In view of the scale invariant nature of the model shown in Figure 1, it is reasonable to anticipate that parallel to molecular dynamics at scale $\beta=\mathrm{m}$ in Eq. (132a) and chromo-dynamics at scale $\beta=\mathrm{k}$ in Eq. (132b), Helmholtz decomposition should also be valid in cosmology at galactic-dynamics scale $\beta=\mathrm{g}$, and in isotropic turbulence at equilibrium eddy-dynamics EED scale $\beta=$ e [39].

The results in equations (132a)-(132b) suggest that in harmony with the concept of dark energy and dark matter in cosmology [99-104] one can express Eq. (131) as sum of dark energy and dark matter

$$
E_{\beta}=\frac{3}{4} \mathrm{~T}_{\beta} S_{\beta}+\frac{1}{4} \mathrm{~T}_{\beta} S_{\beta}=D E_{\beta}+D M_{\beta}
$$

Hence, of the total energy of the field TS, $3 / 4$ is associated with free heat and identified as electromagnetic mass hence dark energy and $1 / 4$ is associated with latent heat and identified as gravitational mass or dark matter [40]. The predicted [33] fractions $3 / 4$ and $1 / 4$ were found to be in close agreement with the recent observations by Riess et al., [105, 106], Schmidt et al., [107], and Perlmutter et al., [108].

The result in Eq. (134) is also in accordance with general theory of relativity [109-110] as described by Pauli [110]

"The energy of a spatially finite universe is three-
quarters electromagnetic and one-quarter
gravitational in origin"

Scale invariance of the model suggests that dark matter in Eq. (134) may be expressed as [33]

$$
D M_{\beta}=E_{\beta-1}=D E_{\beta-1}+D M_{\beta-1}
$$

Therefore, according to Equations (134) and (135) all matter in the universe is composed of dark energy hence electromagnetic mass as anticipated by both Lorentz [97] and Poincaré [68, 89-90].

\section{Concluding remarks}

Some implications of a scale-invariant model of statistical mechanics to classical and relativistic thermodynamics were investigated. Modified transformations for thermodynamic properties were introduced. Helmholtz decomposition of heat into what he called free and latent heat was shown to lead to a modified form of the first law of thermodynamics. Also, modified properties of ideal gas were presented. Subjective versus objective aspects of Boltzmann thermodynamic entropy and Shannon information entropy were discussed. Finally, it was shown that of the total energy constituting matter $3 / 4$ is dark energy and represents electromagnetic mass and $1 / 4$ is dark matter and represents the gravitational mass in accordance with general theory of relativity.

\section{Acknowledgements}

This research was supported by NASA grant No. NAG31863 and in part supported by The U.S. National Science Foundation grant No. CTS-8820077. The author also expresses his gratitude to one reviewer for the constructive comments.

\section{Nomenclature}

$a$ speed of sound $\sqrt{\mathrm{p} / \rho}[\mathrm{m} / \mathrm{s}]$

$\tilde{a}$ speed of sound $\sqrt{\gamma \mathrm{RT}^{\prime}}[\mathrm{m} / \mathrm{s}]$

$a^{\prime}$ speed of sound $\sqrt{\mathrm{p}^{\prime} / \rho}[\mathrm{m} / \mathrm{s}]$

c speed of light $[\mathrm{m} / \mathrm{s}]$

$\mathrm{c}_{\mathrm{p}}$ specific heat at constant pressure [J/kg.K]

$\mathrm{c}_{\mathrm{v}} \quad$ specific heat at constant volume $[\mathrm{J} / \mathrm{kg} . \mathrm{K}]$

$D M$ dark matter [J]

$D E$ dark energy[J]

$E \quad$ energy [J]

$f \quad$ velocity distribution function

f arbitrary function

$F \quad$ Helmholtz free energy [J]

$G \quad$ Gibbs free energy [J]

$\hat{g} \quad$ atomic Gibbs free energy [J/particle]

$g \quad$ specific Gibbs free energy $[\mathrm{J} / \mathrm{kg}]$

$\mathrm{g}$ number of degeneracy

$\mathrm{H}$ Shannon entropy

$H$ enthalpy [J]

$\tilde{h}$ molar absolute enthalpy [J/kmol]

$\hat{h}$ atomic absolute enthalpy [J/particle]

h Planck constant $[\mathrm{j}-\mathrm{s}]$

I information entropy

I moment of inertia $\left[\mathrm{kg} \cdot \mathrm{m}^{2}\right]$

$\mathbf{J}_{\mathrm{c}} \quad$ mechanical equivalent of heat $[\mathrm{J} / \mathrm{kcal}]$

$\mathrm{J}$ modified mechanical equivalent of heat $[\mathrm{J} / \mathrm{kcal}]$

$K \quad$ Shannon constant measure

$\mathrm{k} \quad$ Boltzmann constant $[\mathrm{J} / \mathrm{K}]$

$\mathrm{L}$ system length [m]

$\ell \quad$ atomic free path $[\mathrm{m}]$

$\mathrm{m} \quad$ atomic mass $[\mathrm{kg}]$

$\mathrm{m}_{\mathrm{r}} \quad$ Lorentz relativistic mass $[\mathrm{kg}]$

$\mathrm{m}_{\mathrm{k}}$ photon gravitational mass $[\mathrm{kg}]$

$\mathrm{N}$ number of particles

$\mathrm{N}^{\mathrm{o}} \quad$ Avogadro-Loschmidt number [molecules/mole]

$\overline{\mathrm{p}}_{\beta}$ root-mean-square momentum $\left[\mathrm{N} / \mathrm{m}^{2}\right]$

$\mathrm{p} \quad$ modified pressure $\left[\mathrm{N} / \mathrm{m}^{2}\right]$

$\mathrm{p}^{\prime} \quad$ classical pressure $\mathrm{p}^{\prime}=2 \mathrm{p}\left[\mathrm{N} / \mathrm{m}^{2}\right]$

$p$ probability

$Q \quad$ reversible heat [kcal]

$\mathrm{R}^{\circ} \quad$ universal gas constant [J/kmol.K]

$\mathrm{R}$ gas constant $\mathrm{R}^{\circ} / \tilde{\mathrm{W}} \quad[\mathrm{J} / \mathrm{kg} . \mathrm{K}]$

$S$ entropy [J/K]

$\hat{\mathrm{s}} \quad$ atomic entropy $[\mathrm{J} / \mathrm{K}]$

$\mathrm{T}^{\prime} \quad$ classical absolute temperature $[\mathrm{K}]$

$\mathrm{T}$ modified absolute temperature $\mathrm{T}^{\prime} / 2[\mathrm{~K}]$

$\mathrm{t}$ time $[\mathrm{s}]$

$U \quad$ internal energy [J]

u "atomic" velocity $[\mathrm{m} / \mathrm{s}]$

$\hat{u}$ "atomic" internal energy [J/particle]

$\mathbf{V}$ diffusion velocity [m/s] 
$\mathbf{V}^{\prime}$ peculiar velocity $[\mathrm{m} / \mathrm{s}]$

$V \quad$ volume $\left[\mathrm{m}^{3}\right]$

v element velocity $[\mathrm{m} / \mathrm{s}]$

$\hat{\mathrm{v}}$ "atomic" volume $\left[\mathrm{m}^{3} /\right.$ particle $]$

$\mathrm{W}$ number of complexions

$\overline{\mathrm{W}}$ total number of complexions $\mathrm{W}^{4}$

$\tilde{\mathrm{W}}$ molecular weight $[\mathrm{kg} / \mathrm{kmol}]$

$W$ reversible work $[\mathrm{J}]$

w convective velocity $[\mathrm{m} / \mathrm{s}]$

$x$ space coordinate [m]

$\mathrm{Z}$ partition function

\section{Greek Letters}

$\beta$ index for scale

$\gamma \quad$ specific heat ratio $=\mathrm{c}_{\mathrm{p}} / \mathrm{c}_{\mathrm{v}}$

$\varepsilon \quad$ thermal energy density $=\rho \tilde{h}\left[\mathrm{~J} / \mathrm{m}^{3}\right]$

$\theta$ angular coordinate [rad]

$\lambda$ wavelength [m]

$\lambda_{\mathrm{w}} \quad$ Wien wavelength [m]

$\hat{\mu} \quad$ atomic Gibbs chemical potential [J/particle]

$v$ frequency $[\mathrm{Hz}]=[1 / \mathrm{s}]$

$\rho \quad$ mass density $\left[\mathrm{kg} / \mathrm{m}^{3}\right]$

$\tau$ period [s]

$\chi \quad$ spring constant $\left[\mathrm{J} / \mathrm{m}^{2}\right]$

\section{References}

[1] L. de Broglie, Interference and Corpuscular Light. Nature 118, 2969, 441-442, 1926; Sur la Possibilité de Relier les Phénomènes d'Interférence et de Diffraction à la Théorie des Quanta de Lumière. C. R. Acad. Sci. Paris, 183, 447-448, 1927; La Structure Atomique de la Matière et du Rayonnement et la Mécanique Ondulatoire. 184, 273-274, 1927; Sur le Rôle des Ondes Continues en Mécanique Ondulatoire. 185, 380-382, 1927.

[2] L. de Broglie, Non-Linear Wave Mechanics: A Causal Interpretation, Elsevier, New York, 1960.

[3] L. de Broglie, The Reinterpretation of Wave Mechanics. Found. Phys. 1, 5-15, 1970.

[4] E. Madelung, "Quantentheorie in Hydrodynamischer Form," Z. Physik. 40, 332-326, 1926.

[5] E. Schrödinger, Über die Umkehrung der Naturgesetze, Sitzber Preuss Akad Wiss Phys-Math Kl, 144-153, 1931.

[6] R. Fürth, Über Einige Beziehungen zwischen klassischer Staristik und Quantenmechanik, Z. Phys. 81, 143-162, 1933.

[7] D. Bohm, A Suggested Interpretation of the Quantum Theory in Terms of "Hidden" Variables. I, Phys. Rev. $85,166-179,1952$.

[8] T. Takabayasi, On the Foundation of Quantum Mechanics Associated with Classical Pictures, Prog. Theor. Phys. 8, 143-182, 1952.

[9] D., Bohm, and J. P. Vigier, Model of the Causal Interpretation of Quantum Theory in Terms of a Fluid with Irregular Fluctuations, Phys. Rev. 96, 208-217, 1954.

[10] E. Nelson, Derivation of the Schrödinger Equation from Newtonian Mechanics. Phys. Rev. 150, 1079$1085,1966$.

[11] E. Nelson, Quantum Fluctuations, Princeton University Press, Princeton, New Jersey, 1985.

[12] L. de la Peña, New Foundation of Stochastic Theory of Quantum Mechanics. J. Math. Phys. 10, 1620-1630, 1969.

[13] L. de la Peña, and A. M. Cetto, Does Quantum Mechanics Accept a Stochastic Support? Found. Phys. 12,1017-1037, 1982.

[14] A. O. Barut, Schrödinger's Interpretation of $\psi$ as a Continuous Charge Distribution. Ann. der Phys. 7, 3136, 1988 .

[15] A. O. Barut, and A. J. Bracken, Zitterbewegung and the Internal Geometry of the Electron. Phys. Rev. D 23, 2454-2463, 1981.

[16] J. P. Vigier, De Broglie Waves on Dirac Aether: A Testable Experimental Assumption, Lett. Nuvo Cim. 29, 467-475, 1980; Ph. Gueret, and J. P. Vigier, De Broglie's Wave Particle Duality in the Stochastic Interpretation of Quantum Mechanics: A Testable Physical Assumption, Found. Phys. 12, 1057-1083, 1982; C. Cufaro Petroni, and J. P. Vigier, Dirac's Aether in Relativistic Quantum Mechanics, Found. Phys. 13, 253-286, 1983; J. P. Vigier, Derivation of Inertia Forces from the Einstein-de Broglie-Bohm (E.d.B.B) Causal Stochastic Interpretation of Quantum Mechanics, Found. Phys. 25, 1461-1494, 1995.

[17] F. T. Arecchi, and R. G. Harrison, Instabilities and Chaos in Quantum Optics, Springer-Verlag, Berlin, 1987.

[18] O. Reynolds, On the Dynamical Theory of Incompressible Viscous Fluid and the Determination of the Criterion, Phil. Trans. Roy. Soc. A 186, 23-164, 1895.

[19] D. Enskog, Kinetische Theorie der Vorgange in Massig Verdunnten Gasen, by Almqvist and Wiksells Boktryckeri-A.B., Uppsala, 1917. English translation in Ref. [63], pp.125-225.

[20] G. I. Taylor, Statistical Theory of Turbulence-Parts IIV. Proc. Roy. Soc. A 151, 421-478, 1935.

[21] T. Kármán, and L. Howarth, On the Statistical Theory of Isotropic Turbulence, Proc. Roy. Soc. A 164, 192$215,1938$.

[22] H. P. Robertson, The Invariant Theory of Isotropic Turbulence, Proc. Camb. Phil. Soc. 36, 209-223 1940.

[23] A. N. Kolmogoroff, Local Structure on Turbulence in Incompressible Fluid, C. R. Acad. Sci. U. R. S. S. 30, 301-305, 1941; Dissipation of Energy in Locally Isotropic Turbulence, C. R. Acad. Sci. U. R. S. S. 32, 1921, 1942; A Refinement of Previous Hypothesis Concerning the Local Structure of Turbulence in a Viscous Incompressible Fluid at High Reynolds Number, J. Fluid Mech. 13, 82-85, 1962.

[24] A. M. Obukhov, On the Distribution of Energy in the Spectrum of Turbulent Flow, C. R. Acad. Sci. U. R. S. S. 32, 19-22, 1941; Some Specific Features of Atmospheric Turbulence, J. Fluid Mech. 13, 77-81, 
1962.

[25] S. Chandrasekhar, Stochastic Problems in Physics and Astronomy, Rev. Mod. Phys. 15, 1-89, 1943.

[26] S. Chandrasekhar, Stochastic, Statistical, and Hydrodynamic Problems in Physics and Astronomy, Selected Papers, vol.3, University of Chicago Press, Chicago, 1989, pp. 199-206.

[27] W. Heisenberg, On the Theory of Statistical and Isotropic Turbulence, Proc. Roy. Soc. A 195, 402-406, 1948; Zur Statistischen Theorie der Turbulenz, Z. Phys. 124, 628-657, 1948.

[28] G. K. Batchelor, The Theory of Homogeneous Turbulence, Cambridge University Press, Cambridge, 1953.

[29] L. D. Landau, and E. M. Lifshitz, Fluid Dynamics, Pergamon Press, New York, 1959.

[30] H. Tennekes, and J. L. Lumley, A First Course In Turbulence, MIT Press, 1972.

[31] S. H. Sohrab, "Transport Phenomena and Conservation Equations in Multicomponent Chemically-Reactive Ideal Gas Mixtures," Proceeding of the 31st ASME National Heat Transfer Conference, HTD-Vol. 328, 1996, pp. 37-60.

[32] S. H. Sohrab, A Scale Invariant Model of Statistical Mechanics and Modified Forms of the First and the Second Laws of Thermodynamics, Rev. Gén. Therm. 38, 845-853, 1999.

[33] S. H. Sohrab, "The Nature of Mass, Dark Matter, and Dark Energy in Cosmology and the Foundation of Relativistic Thermodynamics," In: New Aspects of Heat Transfer, Thermal Engineering, and Environment. S. H. Sohrab, H. J. Catrakis, N. Kobasko, (Eds.), WSEAS Press, 2008, pp. 434-442.

[34] S. H. Sohrab, Boltzmann Entropy of Thermodynamics versus Shannon Entropy of Information Theory. Int. J. Mech. 8, 73-84, 2014.

[35] S. H. Sohrab, Invariant Planck Energy Distribution Law and its Connection to the Maxwell-Boltzmann Distribution Function. WSEAS Transactions on Mathematics 6, 254-262, 2007.

[36] S. H. Sohrab, "Derivation of Invariant Forms of Conservation Equations from the Invariant Boltzmann Equation," In: Theoretical and Experimental Aspects of Fluid Mechanics, S. H. Sohrab, H. C. Catrakis, and F. K. Benra (Eds.),WSEAS Press, 2008, pp. 27-35.

[37] S. H. Sohrab, "Universality of a Scale Invariant model of Turbulence and its Quantum Mechanical Foundation," In: Recent Advances in Fluid Mechanics \& Aerodynamics, S. Sohrab, H. Catrakis, and. N. Kobasko (Eds.), WSEAS Press, 2009, pp. 134-140.

[38] S. H. Sohrab, “On a scale invariant model of statistical mechanics and derivation of invariant forms of conservation equations from invariant Boltzmann and Enskog equations,". Proceedings of The 2014 International Conference on Mechanics, Fluid Mechanics, Heat and Mass Transfer, February 22-24, Interlaken, Switzerland, 2014, pp. 19-37.
[39] S. H. Sohrab, Quantum Theory of Fields from Planck to Cosmic Scales, WSEAS Transactions on Mathematics 9, 734-756, 2010.

[40] S. H. Sohrab, "On a Scale Invariant Model of Statistical Mechanics, Kinetic Theory of Ideal Gas, and Riemann Hypothesis,". In: Recent Research in Circuits \& Systems, $16^{\text {th }}$ International Conference on Systems. E. Balas, M. Koksal, and V. Vasek (Eds.), WSEAS Press, 2012, pp. 505-546.

[41] R. S. de Groot, and P. Mazur, Nonequilibrium Thermodynamics, North-Holland, 1962.

[42] H. Schlichting, Boundary-Layer Theory, McGraw Hill, New York, 1968.

[43] F. A. Williams, Combustion Theory, Addison Wesley, New York, 1985.

[44] J. O. Hirschfelder, C. F. Curtiss, and R. B. Bird, Molecular Theory of Gases and Liquids, Wiley, New York, 1954.

[45] S. Chapman, and T. G. Cowling, The Mathematical Theory of Non-uniform Gases, Cambridge University Press, Cambridge, 1953.

[46] B. L. van der Waerden, Towards Quantum Mechanics, in: Sources of Quantum Mechanics, B. L. van der Waerden (Ed.), pp.1-59, Dover, New York, 1967.

[47] H. B. G. Casimir, On the Attraction between Two Perfectly Conducting Plates, Proc. K. Ned. Akad. Wet. 51, 793-795, 1948.

[48] M. Planck, On the Law of the Energy Distribution in the Normal Spectrum, Ann. der Phys. 4, 553-558, 1901.

[49] M. Planck, The Theory of Heat Radiation, Dover, New York, 1959.

[50] J. D. Jackson, Classical Electrodynamics, Wiley, New York, 1975.

[51] I. Newton, Optics, Dover, New York, 1952.

[52] L. de Broglie, Le Mécanique Ondulatoire du Photon 1, Paris: Herman et Cie, 1940.

[53] L. Bass, and E. Schrödinger, Must the Photon Mass be Zero? Proc. Roy. Soc. A 232, 654-661, 1955.

[54] J. P. Vigier, Possible Test of the Reality of Superluminal Phase Waves and Particle Phase Space Motions in the Einstein-de-Broglie-Bohm Causal Stochastic Interpretation of Quantum Mechanics, Found. Phys. 24, 61-83, 1994.

[55] O. Costa. de Beauregard, The Great Vail, Reality, and Louis de Broglie: Personal Memories, in: Waves and Particles in Light and Matter. A. van der Merwe, and A. Garuccio (Eds.), 1-7, Plenum Press, New York, 1994.

[56] M. W. Evans, On Longitudinal Free Space-time Electric and Magnetic Fields in the Einstein-de Broglie Theory of Light, in: Waves and Particles in Light and Matter. A. van der Merwe and A. Garuccio (Eds.), pp. 259-294, Plenum Press, New York, 1994.

[57] R. Clausius, Über die Art der Bewegung, welche wir Wärme nennen. Annalen der Phyzik, 100, 353-80; 1957. Philosophical Magazine 14,108-127, 1957. 
[58] S. H. Sohrab, "Some implications of a scale invariant model of statistical mechanics to classical and relativistic thermodynamics,". In: Recent Researches in Electric and Energy Systems, Ki Young Kim, Dario Assante, Marian Ciontu, and Jana Jirickova (Eds.), WSEAS press, Athens, 2013, pp. 298-313.

[59] G. 't Hooft, Quantum Gravity as a Dissipative Deterministic System, Class. Quantum Grav. 16, 3263 3279, 1999.

[60] R. Clausius, Ueber einen auf die Wärme anwendbaren mechanischen Satz. Sitzungsberichte der Niedderrheinischen Gesellschaft, Bonn, 114-119, 1870.

[61] S. H. Sohrab, Scale-Invariant Form of the Planck Law of Energy Distribution and its Connection to the Maxwell-Boltzmann Distribution, Bull. Amer. Phys. Soc. 49, 255, 2004.

[62] S. Chandrasekhar, Newton's Principia for the Common Reader, 579-593, Oxford University Press, New York, 1995.

[63] G. S. Brush, Kinetic Theory, Vol.1-3, Pergamon Press, New York, 1965.

[64] H. Helmholtz, Über der Ehaltung der Kraft, Eine Physikalische Abhandlung. G. Reiner, Berlin, 1947. English translation in: G. S. Brush, Kinetic Theory, Vol.1-3, Pergamon Press, New York, 1965.

[65] W. Pauli, Pauli Lectures on Physics, Vol.3, p. 14, MIT Press, 1973.

[66] C. A. Long, and S. H. Sohrab, "The Power of Two, Speed of Light, Force and Energy and the Universal Gas Constant,". In: Recent Advances on Applied Mathematics, C. A. Long, S. H. Sohrab, G. Bognar, and L. Perlovsky, (Eds.), WSEAS Press, 2008, pp. 434-442.

[67] O. De Pretto, Ipotesi dell'Etere Nella Vita dell'Universo, Reale, Inst. Veneto di Scienze, Lettere en Arti 63, 439-500, 1904.

[68] H. Poincaré, La Théorie de Lorentz et le Principe de Réaction. Arch. Neerland. 5, 252-278, 1900; http://www.physicsinsights.org/poincare-1900.pdf.

[69] F. Hasenöhrl, Zur Theorie der Strahlung in bewegten Körpern. Ann. der Physik 15, 344-370, 1905. Zur Theorie der Strahlung in bewegten Körpern. Ann. der Phys. 16, 589-592, 1905.

[70] A. Einstein, Ist die Trägheit eines Körpers von seinem Energieinhalt abhängig? Ann. der Phys. (Leipzig) 18, 639-641, 1905.

[71] M. Kardar, Statistical Physics of Particles, Cambridge University Press, New York, 2007.

[72] R. E. Sonntag, and G. E. van Wylen, Fundamentals of Statistical Thermodynamics, Wiley, New York, 1966.

[73] W. Yourgrau, A. van der Merwe, and G. Raw, Treatise on Irreversible and Statistical Thermodynamics, Dover, New York, 1982.

[74] C. E. Shannon, The Mathematical Theory of Communication, Bell System Tech. J. 27, 379-423 and 623-656, 1948.

[75] W. Weaver, and C. E. Shannon, The Mathematical
Theory of Communication, University of Illinois Press, Chicago, 1963.

[76] L. Boltzmann, Weitere Studien uber das Warmegleichgewicht unter Gasmoleculen. Sitzungsberichte Akad.Wiss., Vienna, Part II, 66, 275370, 1872. English translation in: G. S. Brush, Kinetic Theory, Vol.1-3, pp. 88-175, Pergamon Press, New York, 1965.

[77] L. Boltzmann, Lectures on Gas Theory, Dover, New York, 1964.

[78] O. Darrigol, Statistics and Combinatorics in Early Quantum Theory, Historical Studies in the Physical and Biological Sciences, 19, 17-80, 1988; Statistics and Combinatorics in Early Quantum Theory, II: Early Symptoms of Indistinguishability and Holism, 21, 237298, 1991.

[79] L. Brillouin, Maxwell Demon Cannot Operate: Information and Entropy I, J. Appl. Phys. 22, 334-337, 1951.

[80] A. Ben-Naim, Entropy Demystified, World Scientific, New York, 2008.

[81] M. Planck, Where Is Science Going, Ox Bow Press, Connecticut, 1981.

[82] A. Ben-Naim, A Farewell to Entropy: Statistical Thermodynamics Based on Information, World Scientific, New York, 2008.

[83] M. Planck, Zur Dynamik bewegter Systeme, Sitzungsber. Preuss. Akad. Wiss., 542; Ann. Physik 26, $1,1908$.

[84] K. Mosengeil, Theorie der stationären Strahlung in einem gleichförmig bewegten Hohlraum, Ann. Physik 22, 876, 1907.

[85] A. Einstein, Relativitätsprinzip und die aus demselben gezogenen Folgerungen, Jahrb. Radioaktivität und Elektron 5, 411, 1907.

[86] M. Laue, Das Relativitätsprinzip. Friedr. Vieweg \& Sohn, Braunschweig, 1911.

[87] F. Jüttner, Das Maxwellische Gesetz der Geschwindigkeitsverteilung in der Relativtheorie, Ann. Phys., Lyz. 34, 856, 1911.

[88] W. Pauli, Theory of Relativity, p.135, Dover, 1958.

[89] H. Poincaré, Sur la Dynamique de l'Electron, $C$. $R$. Acad. Sci. Paris 140, 1504-1508, 1905.

[90] H. Poincaré, Sur la Dynamique de l'Electron, Rend. Circ. Mat. Palermo 21, 9-175, 1906.

[91] A. Einstein, Zur Elecrodynamik bewegter Körper, Ann. der Phys. (Leipzig) 17, 891-921, 1905.

[92] C. Liu, Einstein and Relativistic Thermodynamics in 1952: a Historical and Critical Study of a Strange Episode in the History of Modern Physics, BJHS 25, 185, 1992.

[93] H. Ott, Lorentz Transformation of Heat and Temperature, Zeitschrift fur Physik. 175, 70, 1963.

[94] W. Schroeder, and H-J. Treder, The "Einstein-Laue" discussion. BJHS 27, 1113, 1992. 
[95] P. T. Landsberg, and G. E. A. Matsas, Laying the Ghost of the Relativistic Temperature Transformation, Phys. Lett. A. 23, 401, 1996.

[96] M. Requardt, Thermodynamics Meets Special Relativity - or what is real in Physics? arXiv:0801.2639 v1[gr-qc], 17 Jan, 2008.

[97] H. Lorentz, Electromagnetic Phenomena in a System Moving with any Velocity less than that of Light, in The Principles of Relativity, Dover, 1952.

[98] I. Avramov, Relativity and Temperature, Russ. J. Phys. Chem. 77, S179, 2003.

[99] A. G. Lemaitre, A Homogeneous Universe of Constant Mass and Growing Radius Accounting for the Radial Velocity of Extragalactic Nebulae, Monthly Notices of the Roy. Astr. Soc. xci, 483,1931; PNAS 20, 12, 1934.

[100] M. S. Turner, Dark Matter in the Universe, Physica Scripta T36, 167, 1991.

[101] L. M. Krauss, and M. S. Turner, The Cosmological Constant is Back. arXiv:astro-ph/9504003 v1 3, Apr, 1995.

[102] M. S. Turner, The case of $\Omega_{\mathrm{M}}=0.35 \pm 0.035$. ApJ. 576, L101, 2002.
[103] Z. G. Dai, E. W. Liang, and D. Xu, Constraining $\Omega_{\mathrm{M}}$ and Dark Energy with Gamma-Ray Bursts, ApJ. 612, L102, 2004.

[104] D. Polarski, Dark Energy: Current Issues, Ann. Physik 15, 342, 2006.

[105] A. G. Riess, et al., Observational Evidence from Supernovae for an Accelerating Universe and a Cosmological Constant, AJ. 116, 1009, 1998.

[106] A. G. Riess, et al., Type Ia Supernova Discoveries at $z>1$ from the Hubble Space Telescope: Evidence for Past Deceleration and Constraints on Dark Energy Evolution, ApJ. 607, 665, 2004.

[107] B. G. Schmidt, et al., The High-Z Supernovae Search: Measuring Cosmic Deceleration and Global Curvature Using Type Ia Supernovae, ApJ. 507, 46, 1998.

[108] S. Perlmutter, et al., Measurements of $\Omega$ and $\Lambda$ from 42 High-Redshift Supernovae, ApJ. 517, 565, 1999.

[109] A. Einstein, Do Gravitational Fields Play an Essential Part in the Structure of the Elementary Particles of Matter, in: The Principles of Relativity, Dover, 1952.

[110] W. Pauli, Theory of Relativity, p. 204, Dover, 1958. 